\title{
SYMPTOME ET DIAGNOSTIC DANS LA CLINIQUE PSYCHANALYTIQUE : ENJEUX EPISTEMIQUES, CLINIQUES, POLITIQUES ET SOCIETAUX
}

Symptom and diagnosis in clinical psychoanalys is: epistemic, clinical, political and societal issues

Síntoma y diagnóstico en la clínica psicoanalítica: cuestiones epistémicas, clínicas, políticas y sociales

Sintoma e diagnóstico na clínica psicanalítica: questões epistêmicas, clínica, políticas e sociais

Sidi Askofaré ${ }^{1}$

Psychanalyste

Professeur des Universités

Université de Toulouse-Jean Jaurès

\section{RÉSUMÉ}

Cet article vise à reprendre la problématique du diagnostic dans une perspective plus large, en essayant d'examiner les enjeux cliniques, épistémiques, éthiques et politiques des incidences du dernier enseignement de Lacan sur la théorie psychanalytique et, en particulier, concernant les formes d'assujettissement aux structures cliniques. On propose 5 points pour examiner ces enjeux: la question clinique générale du diagnostic; les fondements du diagnostic en psychanalyse; la perspective structuraliste sur le diagnostic; l'intégration de la critique du structuralisme; la place de la considération du symptôme. Enfin, on terminera sur un échange autour des enjeux politiques, pour aujourd'hui et le futur proche, de la question diagnostique, notamment dans ses effets de ségrégation et d'exclusion.

Mots-clés: Symptôme, diagnostic, clinique psychanalytique

\section{ABSTRACT}

This article aims to approach the problem of diagnosis in a broader perspective, trying to look in terms of clinical, epistemic, ethical and political issues from the last teaching of Lacan on psychoanalytic theory, especially concerning forms of subjugation for clinical structures. These is sues are considered in five points: general clinical question of diagnosis; the foundations of diagnosis in psychoanalysis; the structuralist pers pective on the diagnosis; integration of criticism of structuralism; the place of the consideration of the symptom. Finally, it ends with an exchange about politic is sues, for today and the near future, on the emission of the diagnostic, including its effects of segregation and exclusion.

Keywords: Symptom, diagnostic, clinical psychoanalys is.

\section{RÉSUMEN}

\footnotetext{
1 sidi.askofare@orange.fr
} 
Este artículo tiene como objetivo tomar el problema del diagnóstico en una perspectiva más amplia, tratando de los aspectos clínicos, epistémicos, éticos y políticos a partir de la última enseñanza de Lacan sobre la teoría psicoanalítica, especialmente en lo concerniente a las formas de imposición de estructuras clínicas. Cuenta con 5 puntos a considerar estas cuestiones: la cuestión clínica general de diagnóstico; las bases del diagnóstico en el psicoanálisis; la perspectiva estructuralista en el diagnóstico; la integración de la crítica del estructuralismo; el lugar de la consideración del síntoma. Por último, se termina con un intercambio sobre cuestiones de política para el presente y el futuro cercano de la emisión del diagnóstico, incluyendo sus efectos de segregación y exclusión.

Palabras-clave: Síntoma; diagnóstico; clínica psicoanalítica

\section{RESUMO}

Este artigo tem por objetivo retomar a problemática do diagnóstico em uma perspectiva mais ampla, tentando examinar questões clínicas, epistêmicas, éticas e políticas a partir das incidências do último ensino de Lacan na teoria psicanalítica, em particular no que concerne às formas de assujeitamento às estruturas clínicas. Propomos cinco pontos para examinar essas questões: a questão clínica geral do diagnóstico; os fundamentos do diagnóstico em psicanálise; a perspectiva estruturalista no diagnóstico; a integração da crítica do estruturalismo; o lugar de consideração do sintoma. Enfim, terminamos com um debate em torno das questões políticas, para o hoje e o futuro próximo, da questão diagnóstica, notadamente em seus efeitos de segregação e exclusão.

Palavras-chave: sintoma, diagnóstico, clínica psicanalítica.

\section{INTRODUÇÃO}

Il est toujours périlleux de s'engager dans la conception et la réalisation d'un projet de Séminaire en ayant aucune idée du savoir auprès duquel on va se représenter comme sujet. Ma venue à Brasilia comportait d'animer un Séminaire sur un sujet, en accord avec mes hôtes, susceptible d'intéresser des chercheurs et des praticiens. Très facilement, je dois dire, nous sommes tombés d'accord sur le thème du diagnostic sur une proposition de Juliano Lagôas.

Juliano avait une idée très précise de l'orientation qu'il voulait que j'imprime à ce travail. En effet, j'ai cru entendre dans sa demande, que ce ne soit pas une énième session de formation technique sur le diagnostic mais l'occasion de reprendre la problématique du diagnostic dans une perspective plus large, en essayant d'examiner notamment ce que $\mathrm{j}$ 'ai pu dès lors formuler en termes d'enjeux cliniques, épistémiques, éthiques et politiques.

$\mathrm{Au}$ fond, la seule chose que j'ai tenu à ajouter c'est le terme de symptôme. D'où le titre terminal: "Symptôme et diagnostic dans la clinique psychanalytique: enjeux cliniques, épistémiques, éthiques et politiques ». Par ce choix, j'avais l'idée de faire valoir un certain nombre de choses qui me tiennent à cœur et qui sont relatives aux incidences du dernier enseignement de Lacan sur la théorie psychanalytique, et en particulier concernant les formes d'assujettissement à la structure (les dites structures cliniques), et du coup le diagnostic dit de structure, la question des suppléances et des solutions inventées par les sujets selon leur génie propre. À ce moment-là, j'étais loin d'imaginer la convergence qui existait entre mes collègues de Brasilia et moi autour de cette question essentielle, centrale du symptôme.

C'est donc avec un plaisir sans mélange que je vais travailler avec vous autour du thème que j'ai annoncé, en espérant que nous pourrons au moins en faire ensemble un premier tour tellement il est large, important et, il faut bien le dire, difficile. Mais, comme vous le savez, un séminaire n'est pas un cours, c'est un dispositif de travail qui est fondé sur l'interlocution et une élaboration collective. Je compte donc aussi sur vous, sur vos questions, vos réflexions et vos contributions fondées sur votre pratique ou vos recherches pour partager nos expériences et produire ensemble un savoir, je l'espère, nouveau. En tout cas, pour chacun d'entre nous. Pour engager ce travail, j'ai fait le pari que le retour et la réflexion sur les principes et les fondements 
de notre pratique ne sont jamais inutiles, ne serait-ce que parce que ça nous distingue et de la meilleure des façons- de ceux qui ont définitivement opté pour le statut de «techniciens du psychisme » (comme on parle des «techniciens de surface », pour les femmes de ménage) et pour qui, diagnostiquer c'est, comme au garage, identifier tout simplement la panne à réparer.

C'est dire que, selon moi, derrière le mot et la pratique du diagnostic, il y a toute la conception que l'on se fait d'un sujet, d'un symptôme, de la souffrance psychique, de sa prise en charge, de son traitement et de la fin de ce traitement. C'est donc déjà une question complexe et difficile.

Il se trouve que dans ce séminaire, la difficulté est rehaussée par le fait qu'il s'agit, non pas du diagnostic en général, mais du diagnostic en tant qu'elle orienté par la question du symptôme entendu dans son nouveau et son « effet révolutionnaire », pour reprendre l'expression de Lacan dont mon ami MarieJean Sauret a fait le titre d'un de ses livres.

Après réflexion, il me semble qu' on peut assez raisonnablement ramener à 5 les points a partir desquels il convient d'examiner la question:

1) La question clinique générale du diagnostic:

$\rightarrow$ Clinique des phénomènes;

$\rightarrow$ Clinique des structures;

$\rightarrow$ Clinique des limites;

$\rightarrow$ Clinique des nouages, dénouages, épissures, suppléances.

2) Quels fondements au diagnostic en psychanalyse? Ces fondements sont-ils techniques ou éthiques? Et si elles sont avant tout éthiques, en quoi?

3) Dans la mesure où la perspective lacanienne est généralement présentée comme structurale voire structuraliste, il convient d'arrêter sur le diagnostic de structure pour en examiner les fondements et les limites.

4) Pourquoi dans l'après-coup de l'enseignement de Lacan, devons intégrer à notre abord du diagnostic, la critique du structuralisme et la prise en compte de l'histoire, des effets des discours et de l'approche non plus seulement logique mais topologique?

5) Dès lors, quelle est la position juste à se faire face au diagnostic? Quelle place la considération du symptôme occupe -t-elle dans cette perspective?

6) Enfin, nous terminerons sur un échange autour des enjeux politiques, pour aujourd'hui et le futur proche, de la question diagnostique, notamment dans ses effets de ségrégation et d'exclusion.

I) Rien qu'à l'énoncé de ces questions, vous voyez bien que tout le temps imparti au stage, d'hier matin à cet après-midi n'aurait pas suffi à présenter et à discuter sérieusement l'intégralité des ces questions.

Je vais y aller donc, non pas à la hache mais, aujourd'hui, à la louche! Au moins sommes-nous sûr qu'avec une louche on ne peut pas assécher un océan!

On peut partir donc d'une question extrêmement simple: pourquoi le diagnostic? Et la décliner en:

$\rightarrow$ Pourquoi diagnostiquons-nous?

$\rightarrow$ Comment diagnostiquons-nous?

$\rightarrow$ Quels sont les fondements et les coordonnées du diagnostic en psychanalyse?

Si je pose ces questions, ce n'est pas simplement pour ménager une progression dans mon exposé ou pour retarder l'exame n de la question qui nous occup: le symptôme. C'est que le diagnostic comme tel pose un problème dans la communauté des cliniciens. Et ce pas simplement parce que nous avons souvent du mal à nous accorder sur les diagnostics que nous posons; il suffit 
de songer à la dispute interminable relative au diagnostic de Sergueï Pankejeff alias «L'homme aux loups » (névrose obsessionnelle versus psychose), à celle de Sidonie Scillag alias « La jeune homosexuelle » (hystérie versus perversion) ou celle du Président Schreber (paranoïa versus schizophrénie). Même les diagnostics de névrose hystérique qui nous semblaient les plus assurés comme ceux établis par Breuer et Freud dans leurs Études sur l'hystérie sont aujourd'hui contestés au profit de la catégorie d'étatslimites. Alors, quels sont-ils ces problèmes? Pour dire vite, je dirai qu'ils sont relatifs à la nécessité clinique, à la légitimité et à l'éthique du diagnostic dans la pratique psychanalytique. La question se pose pour la simple raison que la notion du diagnostic et sa pratique sont d'origine médicale, appartiennent à la démarche médicale. Si la psychanalyse se l'est appropriée, c'est en tant qu'elle est définissable comme «méthode de traitement des troubles névrotiques 》 fondée sur un «procédé d'investigation des processus psychiques ». Vous aurez reconnu les formules de Freud dans « «Psychanalyse » et «Théorie de la libido » (1922)

Il y a donc tout une réflexion à mener sur le mouvement allant du diagnostic médical stricto sensu au diagnostic tel que la psychanalyse le reconsidère et en fait usage. On peut en trouver des éléments tout à fait utiles et précieux chez quelqu'un comme Michel Foucault, notamment dans sa Naissance de la clinique. Pour aujourd'hui, j'ai choisi une voie plus directe. Je partirai de Freud, et tout particulièrement de son petit texte de 1913 intitulé «Le début du traitement », texte qui complète et approfondit celui de 1905: «De la psychothérapie ». Je m'excuse de citer trop longuement Freud, mais ce qu'il dit ici est essentiel par rapport à ce qui nous occupe qu'il serait dommage de simplement l'évoquer ou le paraphraser: «Il y a plusieurs années déjà, j'ai donné ailleurs de très importantes indications sur le choix des malades. (...) Ces indications ont, entre- temps, été approuvées par d'autres analystes. Toutefois je tiens à ajouter que j'ai maintenant pris l'habitude lorsque je connais encore peu un malade, de pratiquer d'abord un traitement d'essai de une à deux semaines. Si l'on vient à interrompre le traitement au bout de ce temps, on épargne au malade l'impression pénible que lui laisserait l'échec d'une tentative de cure. On se borne, de cette façon, à effectuer un sondage permettant de mieux connaitre le cas et de décider s'il se prête ou non à une psychanalyse. Il n'existe d'ailleurs aucune autre sorte d'épreuve possible. Les entretiens, même fréquents et prolongés, les interrogatoires au cours de consultations ordinaires, ne la remplaceraient pas. Cet essai préliminaire constitue pourtant déjà le début d'une analyse et doit se conformer aux règles qui la régissent; la seule différence peut être que le psychanalys te laisse surtout parler le patient sans commenter ses dires plus qu'il n'est nécessaire à la poursuite de son récit.

Ce court traitement préliminaire présente aussi l'avantage de faciliter le diagnostic. On est souvent obligé de se demander, lorsqu'on a affaire à une névrose avec symptômes hystériques et obsessionnels peu marqués et de courte durée ( cas qu'on serait justement tenté de considérer comme accessible au traitement), si l'on n'a pas affaire à un début de démence précoce, suivant le nom qu'on lui a donné (de schizophrénie, suivant Bleuler ; de paraphrénie, comme je préfère l'appeler) et également si l'on ne risque pas de voir surgir, à un moment donné, des symptômes précis de cette dernière maladie. Je conteste qu'il soit toujours facile d'établir une discrimination. Je sais que certains psychiatres hésitent moins que moi à faire un diagnostic différentiel, mais j'ai pu me convaincre qu'ils se trompent tout aussi souvent. Toutefois il faut noter que pour le psychanalyste l'erreur comporte plus de conséquences fâcheuses que pour le soi-disant psychiatre clinicien. En aucun cas, en effet, ce dernier n'entreprend, quel 
que soit le cas, quelque chose de vraiment efficace, le seul risque qu'il court est de commettre une erreur théorique et son diagnostic ne présente qu'un intérêt académique. $\mathrm{Au}$ contraire dans un cas difficile, le psychanalyste a commis une bévue d'ordre pratique, en causant beaucoup de dépenses inutiles, il discrédite sa méthode de traitement. Quand le patient est atteint non d'hystérie ou de névrose obsessionnelle mais de paraphrénie (schizophrénie), le médecin est dans l'impossibilité de tenir sa promesse de guérison et voilà pourquoi il a tout intérêt à éviter l'erreur de diagnostic. En pratiquant un traitement d'essai de quelques semaines, certains doutes peuvent quelquefo is déterminer le psychanalyste à ne pas poursuivre le traitement. Malheureusement, je ne saurais affirmer que celle-ci permette toujours de se former un jugement sûr, néanmoins elle constitue une utile précaution de plus.

De longs entretiens précédant le début du traitement analytique, une méthode thérapeutique différente antérieurement appliquée, ainsi que des relations amicales déjà établies entre l'analyste et son futur patient, peuvent avoir certaines fâcheuses conséquences auxquelles il faut être préparé. Au moment où le patient commence son analyse, le transfert est déjà établi et le médecin se voit alors contraint de le démasquer lentement au lieu d'être en mesure de le voir naître et croître sous ses yeux, à partir du début du traitement. Le patient conserve ainsi pendant un certain temps une avance qu'on ne lui accorde qu'à contrecœur en analyse»» (La technique psychanalytique, 81-83).

Pour ainsi dire, Freud répond à toutes les questions que j'ai posées et auxquelles nous devions répondre. D'une part le diagnostic est nécessaire en tant qu'il est ce par quoi se décide pour l'analys te l'acte d'engager ou non un sujet dans une analyse. Cette conception est sans doute dépendante de l'idée de Freud, qui n'est plus aujourd'hui la nôtre, idée selon laquelle la psychanalyse serait un traitement portant exclusivement sur les symptômes névrotiques et qui serait, a contrario, sans effets dans les cas de psychoses notamment schizophréniques. Mais même à ne pas être d'accord cette idée de Freud, on ne peut pas ignorer qu'une cure ou un traitement ne saurait conduire de la même manière selon que le patient est névrosé ou psychotique. Il y a donc de ce fait même une nécessité voire une obligation de poser un diagnostic, de forger une hypothèse diagnostique dont dépendra en partie la direction du traitement. D'autre part, ce diagnostic est légitime en ce qu'il ne s'agit pas d'un pur et simple exercice académique, d'un acte de pure prestance relevant du discours universitaire, c'est-à-dire d'une mise en fonction du savoir comme semblant. Il doit être subordonné à la politique et à l'éthique $\mathrm{du}$ discours analytique au même titre que l'interprétation, par exemple. Tombe donc la réponse à la question: pourquoi le diagnostic? Réponse: en vue de la direction de la cure qui requiert que l'analyse ait une petite idée sur la structure et la position du sujet.

Une telle réponse nous indique d'emblée que ce diagnostic analytique que nous appelons aussi «diagnostic de structure » est sans commune mesure avec le diagnostic psychiatrique. Sur quoi est-il fondé ce diagnostic de structure? Je dirai, pour aller vite, qu'il doit être fondé $a$ minima sur trois piliers: la parole, l'hypothèse du sujet et le transfert (à entendre ici en un sens restreint, celui du rapport du sujet à l'Autre). Sa fonction, quant à elle, est de mettre au jour la structure et le type clinique dont relève le sujet. Ce qui suppose bien évidemment d'avoir été formé à ce qu'est la structure et aux différents modes d'assujettissement à ladite structure (névrose, psychose, perversion).

II) C'est à partir de ce point que se pose la question de ce qu'il en est des rapports du diagnostic et de l'éthique.

Ce terme d'éthique, je l'utilise et je pense qu'il convient de l'utiliser, de le 
manier avec beaucoup d'économie, de tact et de délicatesse. D'une part parce que l'éthique n'est pas la morale et encore moins la «moraline », pour parler comme Nietzsche, et d'autre part parce qu'il faut éviter d'en user comme d'un signifiant maitre pour disqualifier l'autre -celui qui ne pense pas ou ne pratique pas comme nous et s'auto-promouvoir. Bref, il convient de ne pas utiliser ce terme comme un " cachemisère », pour reprendre l'expression de $\mathrm{C}$. Castoriadis.

Ceci dit, il y a une dimension éthique dans la mesure où les catégories diagnostiques que nous utilisons impliquent toujours - que nous le sachions ou pas d'ailleurs -des évaluations éthiques. Autrement dit, un acte diagnostique n'est pas réductible à un pur et simple repérage nosographique; il implique toujours le point de perspective d'où le symptôme est évalué. Et ce point de perspective n'est pas le même selon qu'on se situe à partir de la psychopathologie ou à partir de la psychanalyse, mais elle peut varier au sein de la psychanalyse elle-même selon l'idée que l'on se fait de la névrose, de la psychose ou de la perversion, selon le concept qu'on s'est formé du symptôme, du délire ou de l'acte. En atteste le fait que ce point de perspective a pu changer chez Freud mais aussi et surtout chez Lacan comme Colette Soler a pu le rappeler au début de son cours de 2003-2004 sur La querelle des diagnostics.

Parlant de Lacan, je la cite, « on peut suivre, par exemple, qu'au début il a beaucoup de sympathie pour le névrosé et il en parle comme d'une victime émouvante (c'est gentil!), c'est-à-dire qu'il voit dans les névrosés des sujets qui sont en diffic ulté (c'est le cas) mais qui, par là même, puisqu'ils sont en difficulté - vertu du symptôme, donc- révèlent que le désir luimême implique des difficultés. Ce n'est pas eux seulement qui sont en difficulté, le désir lui-même véhicule des difficultés structurales et non seulement ils les révèlent, dit Lacan, mais ils en tiennent compte; et il ajoute - voilà le jugement éthique- ça ne les place pas si mal dans l'échelle humaine.

(...) Mais Lacan qui valorisait le symptôme névrotique tant qu'il était en train d'élaborer la dimension du désir dans l'expérience, fait une autre évaluation quand il commence à s'intéresser à ce qui n'est pas le désir mais la jouissance, et reprend un terme que Freud a souligné dans la parole de l'Homme aux rats, le terme de lâcheté, de couardise. C'est dire que le névrosé, du fait du refoulement, du fait de la défense et tuttiquanti, recule quand il s'agit de reconnaître et d'assumer sa propre jouissance. Et du coup dans ces années-là, on est en '67, il fait compliment au pervers qui, dit-il, «affronte de beaucoup plus près l'impasse sexuelle ».

Elle en conclut - je vous le fait court! - que l'éthique du diagnostic n'est pas la même dans la psychanalyse et dans la psychiatrie. Ce n'est pas étonnant. Pour le psychiatre en tant qu'agent de la santé mentale, les diagnostics, peu ou prou, en dernière analyse, réfèrent toujours à l'adaptation sociale, à la dangerosité ou pas. »

Inutile de dire que ce n'est pas de cela qu'il s'agit pour nous, en psychanalyse, que ce soit en clinique adulte ou a fortiori en clinique de l'enfant. En effet le devoir analytique et l'acte analytique sont autres. Ils visent à ce que le sujet se retrouve dans la structure, dans l'inconscient comme savoir qui le détermine, dans la promotion d'un autre usage de son symptôme au-delà de son sens et du repérage de sa cause.

Tout ceci serait presque facile si les choses ne s'étaient pas plus complexifier depuis l'avènement de la psychanalyse. Je ne vais pas trop m'y étendre, mais il convient de prendre acte du fait que quelque chose a changé dans la manière de considérer et d'envisager le diagnostic. A cela, il y a plusieurs raisons:

$\rightarrow \quad$ Le recul et l'éclatement des grandes nosographies «nationales », avec comme conséquence l'absence 
de références psychopathologiques partagées;

$\rightarrow \quad$ Parallèlement, on assiste à une montée en puissance d'un espéranto prétendument a-théorique, le DSM, qui fait du diagnostic le serviteur de la statistique et de l'épidémiologie.

Par ailleurs, nous observons une multiplication des syndromes et une promotion d'«entités symptomatiques nouvelles » accompagnées d'une mise en question voire d'une mise en cause des structures freudiennes classiques héritées de la nosographie psychiatriques, au profit notamment de la catégorie d'états-limites, de borderline, de personnalités narcissiques et tuttiquanti.

III) Sur le fond de ces considérations générales sur le diagnostic, comment situer alors la question de l'enfant, et donc et celle de la spécificité éventuelle du diagnostic en clinique de l'enfant?

Je vais prendre mon départ dans une thèse massive que vous connaissez bien: dans l'expérience psychanalytique, nous avons à connaître du symptôme au moins sous trois figures:

1) Le symptôme que le sujet est: le sujet en tant que symptôme de l'Autre donc, que cet Autre soit la mère ou le couple parental;

2) Le symptôme que le sujet a, dont il est le porteur et le patient, symptôme auquel il croit («il y croit, croit qu'il veut dire quelque chose », Lacan, R.S.I), symptôme qu'il propose au déchiffrement et dont il attend le traitement (allègement, réduction, disparition) par l'analyse;

3) Le symptôme que le sujet croit (il le croit, croit ce qu'il dit vs il y croit) mais qui peut s'avérer le symptôme qui traite le sujet, la «suppléance » qui assure le nouage des éléments - R, S, I - de la structure en tant que quatrième rond.
C'est dire si la question du symptôme concerne et traverse de part en part la clinique, notamment psychanalytique. C'est dire aussi combien nos pratiques cliniques les plus quotidiennes sont déterminées et orientées par notre conception du symptôme. C'est dire enfin la nécessité et l'urgence qu'il y a à mette au jour et à préciser cette conception pour en tirer les conséquences quant à l'accueil et au traitement du symptôme.

Le plus difficile, vous l'aurez sans doute anticipé ce n'est pas tant de parler du symptôme - aujourd'hui en passe d'être supplanté, y compris chez certains psychanalystes par les notions d'états, de phénomènes, de troubles, de conduites ou de dysfonctionnements que d'en parler de manière juste, c'està-dire d'une manière qui n'en écrase ni les variétés phénoménales, ni les spécificités structurales, ni les complexités conceptuelles ; une manière aussi qui articule et tresse médecine psychiatrique, psychanalyse freudienne et option lacanienne dans le champ freudien.

J'ai repéré dans Lacan « oiseau rare » - il s'agit d'un passage, un peu long également - de son «Introduction à l'édition allemande des Écrits », et je vous propose d'en partir:

«(...) la question commence à partir de ceci qu'il y a des types de symptôme, qu'il y a une clinique Seulement voilà: elle est d'avant le discours analytique, et si celui-ci y apporte une lumière, c'est sûr mais pas certain. Or nous avons besoin de la certitude parce qu'elle seule peut se transmettre de se démontrer. C'est l'exigence dont l'histoire montre à notre stupeur qu'elle a été formulée bien avant que la science y réponde, et que même si la réponse a été bien autre que le frayage que l'exigence avait produit, la condition dont elle partait, soit que la certitude en fût transmissible, y a été satisfaite. 
(...)

Que les types cliniques relèvent de la structure voilà qui peut déjà s'écrire quoique non sans flottement. Ce n'est certain et transmissible que du discours hystérique. C'est même en quoi s'y manifeste un réel proche du discours scientifique. On remarquera que j'ai parlé du réel, et pas de la nature.

Par où j'indique que ce qui relève de la même structure, n'a pas forcément le même sens. C'est en cela qu'il n'y a d'analyse que du particulier: ce n'est pas du tout d'un sens unique que procède une même structure, et surtout pas quand elle atteint au discours.

Il n'y a pas de sens commun de l'hystérique, et ce dont joue chez eux ou elles l'identification, c'est la structure, et non le sens comme ça se lit si bien au fait qu'elle porte sur le désir, c'est-àdire sur le manque pris comme objet; (Cf. le rêve de la belle bouchère - dans la Traumdeutung - devenu par mes soins exemplaire. Je ne prodigue pas les exemples, mais quand je m'en mêle, je les porte au paradigme).

Les sujets d'un type sont donc sans utilité pour les autres d'un même type. Et il est concevable qu'un obsessionnel ne puisse donner le moindre sens au discours d'un autre obsessionnel. C'est même de là que partent les guerres de religion: s'il est vrai que pour la religion (car c'est le seul trait dont elles font classe, au reste insuffisant), il y a de l'obsession dans le coup.

C'est de là que résulte qu'il n'y a communication dans l'analyse que par une voie qui transcende le sens, celle qui procède de la supposition d'un sujet au savoir inconscient, soit au chiffrage. Ce que j'ai articulé: du sujet supposé savoir. » (Autres écrits, 556-557)

A partir de là, plusieurs voies s'ouvrent et plusieurs choix sont possibles. Je distinguerai au moins les cas où la structure est manifeste, où la question du diagnostic différentiel ne se pose pas. Pour en rester à nos références cliniques communes, c'est le cas de Dora, Hans ou L'homme aux rats.

Mais il existe aussi d'autres dans la clinique des situations dans lesquelles la question du diagnostic ne se situe pas au premier plan; autrement dit, il y a des urgences subjectives, des «cas d'urgence » pour reprendre l'expression de Lacan («Préface à l'édition angla ise du Séminaire XI ») qu'il s'agit de peser pour être sûr de pouvoir les satisfaire. Ce qu'il en est du diagnostic ne se posera que par après.

Deuxième observation: c'est que même à faire retour sur ce type de situation pour tenter une hypothèse diagnostique - à mon sens, il n'y a d'ailleurs que ça: tout diagnostic, en psychanalyse, est hypothèse diagnostique, ce qui lui enlève toute sa dimension éventuelle de maitrise - on n'est jamais à l'abri du surgissement d'un matériel qui viendrait remanier l'ordonnancement des éléments sur lesquels on s'est appuyé pour formuler ledit diagnostic.

Troisième observation: c'est que même dans ce cas, il se pourrait que la problématique du symptôme soit dans un retrait tel que le symptôme comme tel ne puisse pas constituer d'emblée le repère diagnostique principal. Cela peut faire comprendre d'ailleurs pourquoi Lacan a été amené, vers la fin, à être direct, à ne pas trop s'embarrasser par rapport à la question du symptôme face à ses analysants: «Il s'agit de les (ses patients) faire entrer par la porte, que l'analyse soit un seuil, qu'il y ait pour eux une véritable demande. Cette demande: qu'est ce dont ils veulent être débarrassés? Un symptôme » («Entretiens dans les universités nord-américaines »; Yale University, 24 novembre 1975, Scilicet, 1976, n 6/7, p. 32)

Nous sommes donc reconduits à la question du diagnostic différentiel mais en tant qu'il est relatif au dispositif clinique dans lequel il s'inscrit: entretiens 
préliminaires, entretien clinique de «présentation de malade » voire contrôle et supervision. Or, à considérer la place du symptôme et sa fonction dans le diagnostic nous sommes conduit très vite à devoir nous poser ou nous re - poser les questions: 1) existe-t-il dans notre champ des symptômes pathognomoniques - (symptôme qui se rencontre seulement dans une maladie déterminée et qui suffit à en établir le diagnostic) -d'une structure ou d'un type clinique? 2) existe-t-il des symptômes transcliniques? Comment faire le départ entre les symptômes et les simples comportements, conduites ou états subjectifs? 3) d'autres phénomènes cliniques comme l'inhibition ou l'angoisse peuvent-ils avoir pour tel sujet une valeur symptomatique? 4) les symptômes non analytiques, les symptômes non constitués peuvent-ils servir de repères en vue d'un diagnostic de structure?

Et ceci, vous l'aurez compris, vaut pour clinique du sujet, c'est-à-dire est indépendant de toute considération relative à l'âge de la vie.

Est-ce à dire qu'il n'y a pas de particularités, de spécificités du diagnostic en clinique de l'enfant?

IV) Pour répondre à cette question, je vais prendre l'envisager au plus simple, en rappelant que la notion de diagnostic différentiel en psychanalyse comporte l'idée de trancher sur l'assujettissement du sujet à une des trois grandes structures cliniques. Naturellement, le psychologue clinicien, notamment lorsqu'il travaille en institution (de l'hôpital psychiatrique aux structures plus souples comme, en France, les CMP, les CMPP ou les CAT) a affaire à un éventail de pathologies plus large que ces trois structures, en raison notamment de types cliniques comme les démences, les épilepsies, les oligophrénies, etc.

Dans la clinique analytique et dans toutes les pratiques cliniques qui ordonnent leur acte à son savoir et à son éthique, le diagnostic vise, en même temps que la structure clinique, la position du sujet en tant que c'est le sujet qui en question dans l'expérience.

En vérité, la difficulté de l'établissement d'un diagnostic tient au paradoxe suivant: ce diagnostic est nécessaire au départ de l'expérience dans la mesure où pour l'essentiel la direction de la cure en dépend. C'est même pour cela que Freud a introduit l'analyse à l'essai et que Lacan a systématisé et formalisé les entretiens préliminaires qui ont pour objectifs:

$\rightarrow$ De cerner la demande du sujet;

$\rightarrow$ De faire une première exploration permettant d'identifier les symptômes, les questions du sujet (sexe, mort, père, existence, etc.) et son rapport à l'acte;

$\rightarrow$ D'obtenir une «rectification subjective », une implication subjective du patient;

$\rightarrow$ De repérer les rapports du sujet à l'Autre et la mise en fonction du sujet supposé savoir;

Mais dans le même temps, et c'est pour cela qu'il y a paradoxe, nous savons que le mode véritable d'assujettissement du sujet à la structure ne se dévoile réellement que dans le transfert. Parfois, il faut bien le reconnaître, même ce repérage peut s'avérer insuffisant, ce qui peut expliquer des déclenchements tardifs de psychoses bien des années après le début d'une analyse.

$\mathrm{Si}$ 'on s'en tient donc à la perspective psychanalytique du diagnostic différentiel, il est exigible, pour chaque cas, de procéder au moins théoriquement en deux étapes:

1) La première consiste à poser un diagnostic négatif. En effet, il est généralement plus facile de dire ce qu'une chose n'est pas plutôt que ce qu'elle est. On peut donc faire, selon la manière dont se présente le cas:

$\rightarrow$ Un diagnostic négatif de psychose, en recherchant méthodiquement et en 
mettant au jour tous les éléments qui contredisent l'hypothèse de la psychose; $\rightarrow$ Ou un diagnostic négatif de névrose, en rassemblant et en ordonnant tous les éléments qui contreviennent à l'hypothèse de la névrose.

Ce premier temps correspond à une véritable investigation de la part de l'analyste; c'est même pourquoi, me semble-t-il, Lacan a réveillé en psychanalyse ce terme d'entretien qui $y$ était fort peu usité. Pour aller vite, je dirai que cette investigation - qui n'engage pas encore, à ce stade, le sujet dans l'association libre -, cette investigation donc, se doit de tourner autour des rapports du sujet à l'Autre et à la jouissance:

$\rightarrow$ Le rapport au signifiant, le mode d'énonciation, le rapport aux énoncés: croyance, doute, certitude, questionnement, oublis, division, suture, etc;

$\rightarrow$ Le rapport du sujet à l'Autre, et notamment aux Autres familiaux. De ce point de vue, le rapport au père (à l'instance paternelle, la loi, l'autorité...), les traces d'une névrose infantile, le «passage adolescent », les conditions et les incidences de la rencontre avec l'Autre sexe, donnent souvent des indications tout à fait cruciales;

$\rightarrow$ Le rapport du sujet à la jouissance et à ses modes de retour aussi bien qu'elle soit intrusive et persécutrice ou silencieusement réglé par le plusde-jouir en fonction dans le fantasme.

L'autre repère important concerne les symptômes justement, symptômes dont la structure nous enseigne toujours sur la structure clinique même s'il faut éviter de le prendre comme son pur et simple reflet.

Or, c'est peut-être là que se pose la spécificité de la clinique de l'enfant.

L'enfant est certes un sujet - et rien de ce qui concerne le sujet ne lui est étranger
-, mais il n'est pas que ça. Comme enfant, il est aussi un objet (de ses parents sans doute, mais aussi parfois, au-delà d'eux, de l'école, des services sociaux, d'autres figures de l'Autre, etc.)

En conséquence de quoi, l'accueil de l'enfant comme son traitement ne peuvent se concevoir indépendamment de ces autres vis-à-vis desquels sa dépendance n'est pas simplement symbolique ou imaginaire. Vous voyez bien à quoi je fais allusion, cette clinique étant plus quotidiennement la vôtre que la mienne.

D'ailleurs, il faut bien voir que cela ne dévalue pas son côté sujet; ça accentue au contraire quelque chose que l'on oublie souvent, à savoir que le sujet entendu au sens de la psychanalyse est loin d'être une monade leibnizienne, que c'est avant tout un «être de relation » (cf. le schéma de la structure du sujet). En termes courants, nous dirons que c'est un fils ou une fille. Et on est fils ou fille que de ..., pas de causa sui, en la matière. Sans compter qu'il n'y a d'analyse que du fils ou de la fille, ou si vous préférez, en termes techniques que de la névrose infantile et de la névrose de transfert.

Par ailleurs, on ne peut pas ignorer ou écarter d'un revers de la main, la question complexe du temps que l'alliance «objective » entre une certaine débilité «développementaliste » et un certain «fondamentalisme structuraliste » a conduit à minorer et à occulter.

Enfin, il y a toute une dimension, celle dite de l'environnement - attention: je ne vire pas écolo! - epuis Winnicott à quoi il convient de donner sa place notamment lorsque les conditions sociales (chômage, pauvreté, alcoolisme, violences familiales, etc.) en viennent à couvrir et à oblitérer les indicateurs de la structure.

Je conclus.

Nous n'en sommes plus aujourd'hui à la critique et au rejet du diagnostic en raison de ce qu'il peut comporter de «violence symbolique », de ravalement ou de stigmatisation. Tout simplement, parce que toute l'histoire de la psychanalyse et des 
pratiques connexes est là pour témoigner du fait que tant au plan épistémique qu'au plan pratique, nous avons besoin de poser des diagnostics. Ne serait-ce que pour nous guider, nous orienter dans notre action qu'elle soit individuelle ou collective dans les cas de travail en équipe. Cela ne veut pas dire, bien sûr, qu'il n'existe pas d'abus en matière de diagnostic. Mais cet abus est relatif à ce que Colette Soler appelait « la prédication éthique et ce qui inspire le jugement éthique interne au diagnostic ». Tout le monde sait ce que des termes comme «pervers », «caractériels » ou même «délirant » peuvent produire comme effets dans les rapports d'une équipe avec un sujet.

Ce qu'il s'agit dès lors d'élaborer pur soi et pour un usage dans le collectif, c'est quelle est la position juste à se forger par rapport au diagnostic. Pour simplifier, je dirai: ni rejet ni fétichisation. Pas de rejet, parce qu'un tel rejet, le plus souvent, n'est pas sans affinités avec la passion de l'ignorance et l'irresponsabilité qui lui fait cortège. Pas de fétichisme non plus, parce que ce serait tendanciellement faire d'une catégorie nosographique la vérité voire l'être d'un sujet. Ce qui peut conduire, si l'on n'y prend garde, à y lire le destin d'un sujet. Alors même que tout ce que la psychanalyse nous enseigne va là contre. En effet, il y a la structure, et il y a le sujet qui cette structure l'habite et en fait quelque chose. C'est ce sujet qui, dans la clinique, est notre affaire. C'est lui que nous avons la charge d'accompagner pour qu'il se repère dans la structure et dans la sienne en particulier. De telle sorte que je suis tenté de dire que la formulation du diagnostic est essentiellement une hypothèse pour diriger un travail, c'est-à-dire pour déterminer la nécessité et les formes d'intervention du clinicien par rapport

$\rightarrow$ A la rectification subjective;

$\rightarrow$ Au transfert;

$\rightarrow$ A l'interprétation, $\rightarrow$ A la « fin $»$ du travail pour ne pas dire à la «fin de l'analyse ».

La question diagnostique, encore... Enjeux épistémiques et cliniques. La névrose, contemporaine?

Pour poursuivre le travail que nous avons commencé ce matin, je vous propose de partir de ce qui nous parait le plus simple, le plus familier, ne serait-ce que parce qu'elle a constitué le sol natif de la clinique psychanalytique, à savoir la névrose.

Il est non seulement utile mais indispensable de partir de la névrose, ne serait-ce qu'en raison de sa disparition tendancielle dans la novlangue psychopathologique qui semble lui préférer les notions qui ont été moins «contaminées » par la découverte freudienne de l'inconscient: troubles, désordres, dysfonctionnements, syndromes, etc.

Partir de la névrose..., oui, mais comment?

Comme je veux rester fidèle à ma perspective problématique, je vous propose de prendre notre départ dans des «cas» cliniques dont on peut dire justement qu'ils sont «problématiques », des cas pour lesquels le diagnostic n'a pas été en tous points évident pour la communauté analytique, des cas qui nous conduisent à poser à nouveaux frais la question: au fond, qu'appelle-t-on névrose?

Le plus prestigieux de ce type de cas demeure sans doute, à mon sens, celui de Serguei Pankejeff alias l'Homme aux loups.

\section{1) «L'Homme aux loups. »}

Rassurez-vous, je ne vais pas être long. Vous vous souvenez sans qu'il s'agit d'un jeune homme de nationalité russe, âgé de de vingt-deux ans, que Freud traita entre 1910 et 1914. Quand il arrive chez Freud, il venait d'hériter de l'immense fortune de son père, décédé deux ans auparavant. 
Quelques années auparavant, à l'âge de dix-huit ans, la santé mentale de SP s'était effondrée à la suite d'une blennorragie. Son adolescence s'était déroulée normalement et il avait achevé ses études secondaires. Mais son enfance avait été dominée par de graves troubles psychiques qui s'étaient déclenchés avant son quatrième anniversaire sous la forme d'une phobie d'animaux, puis s'étaient transformés en - l'expression est de Freuden «névrose obsessionnelle à contenu religieux ».

Entre dix-huit et vingt-deux ans, le patient séjourna dans des sanatoriums en Allemagne, avec le diagnostic d'état maniaco-dépressif (affection dont son père a souffert).

Freud s'est attaché à traiter uniquement les séquelles de la «névrose infantile »d'allure obsessionnelle.

A l'époque de sa publication, l'intérêt de ce cas, aux yeux de Freud, était justement, d'une part, de mettre en évidence l'importance de la vie sexuelle infantile dans la genèse des névroses, et d'autre part de discuter sur le statut réel ou fantasmatique des événements rapportés par le patient relativement à cette période infantile.

A dire vrai, toute l'analyse, avec Freud, est centrée autour d'un rêve qu'enfant SP fit quelques jours avant le Noël qui marquait son quatrième anniversaire. C'est le fameux rêve des loups: l'enfant rêve qu'il est dans son lit, soudain la fenêtre s'ouvre et il voit sur les branches d'un grand noyer face à la fenêtre six ou sept loups blancs avec de grandes queues de renard. Terrifié à l'idée d'être dévoré par les loups, l'enfant crie et s'éveille.

Par un travail d'associations d'idées, Freud va rapprocher ce rêve de la vision réelle, par l'enfant, d'une relation sexuelle de ses parents lorsqu'il avait un an et demi. Cette «scène primitive » était un coït $a$ tergo au cours duquel il a vu son père dressé et sa mère courbée, tous deux vêtus de blanc. Les loups terrifiants représentaient donc le père et son sexe dressé. La scène primitive, réalité ou fantasme, tel est l'objet de la discussion de ce rêve.

Tout le matériel clinique, qui a permis de reconstituer la «névrose infantile », a été livré par SP au cours des six derniers mois de la cure. En effet, comme vous le savez sans doute, dans le but de forcer les résistances et la «passivité » de son patient, Freud avait fixé un terme prématuré à cette analyse qui durait depuis quatre ans - une éternité pour Freud! - Sans résultat. SP, considéré comme guéri, retourna donc en Russie en 1914. Mais la guerre mondiale et la Révolution bolchévique de 1917 le ruinèrent. Les symptômes reprirent sous la forme d'une constipation opiniâtre. Il revint à Vienne, mais ne pouvait plus payer une cure avec Freud. Ce dernier organisa alors une collecte auprès de la Société de psychanalyse pour verser pendant six ans une rente à Serguéi Pankejeff en dédommagement de sa contribution à la théorie psychanalytique.

Vous n'êtes pas sans savoir que très tard, en 1926, Freud reviendra sur ce cas, dans son Inhibition, symptôme, angoisse. Je n'en relèverai que deux passages qui me semblent pouvoir enrichir notre recherche:

a) «L'histoire de la maladie de «l'homme aux loups » russe plaide très nettement en faveur de la dernière possibilité, la plus grave, dégradation régressive réelle dans le ça de la motion orientée vers la génitalité » vs «substitution au représentant d'une expression régressive » - car, à partir du rêve décisif, il se comporte d'une manière «méchante », tourmenteuse, sadique, et développe bientôt là-dessus une authentique névrose obsessionnelle. » (P. 24);

b) «Dans le cas du petit Hans, certaines différences... p. 25-27).

Mais son état s'aggravant, Freud l'adressa à une de ses analysantes, Ruth 
Mack Brunswick, pour un traitement gratuit. Pendant cette période, il souffrait d'un délire hypocondriaque centré sur l'intestin, puis sur le nez et les dents. Ruth M. Brunswick établit quant à elle un diagnostic de paranoïa. Au bout de quelques mois de cure l'état de SP s'améliora sans qu'aucun élément nouveau sur le matériel infantile ne fût livré. Il s'agissait principalement de résoudre le transfert du patient avec Freud, ce qui n'avait pu se faire au moment voulu à cause de l'échéance fixée par ce dernier. Ce forçage de la part de Freud, alliée à l'erreur de la rente versée au patient et aux circonstances douloureuses de sa vie ont précipité une rechute, le déclenchement d'un «épisode psychotique »(Lacan).

2)

3) «L'enfant aux poules »

4) «Perversion sexuelle transitoire au cours d'un traitement psychanalytique »

Si j'ai convoqué ces cas, c'est bien parce que nous avons besoin, pour bien penser ce qu'est la névrose, aussi bien des cas paradigmatiques - comme «Dora», «L'Homme aux rats » ou «Le Petit Hans » - que des cas qui posent plus radicalement la question de ce qui distingue fondamentalement la névrose de la psychose, d'une part, et de la perversion, d'autre part. Si cela est nécessaire, aujourd'hui cela me parait insuffisant. Pourquoi?

Tout simplement parce que nous ne pouvons pas ignorer qu'une grande partie de la communauté psychanalytique a été amenée à promouvoir ce qu'ils appellent les «états-limites », les «borderline » ou les «personnalités narcissiques ».

Je sais que le mouvement sinon «naturel», en tout cas spontané chez les «lacaniens » est de récuser l'existence de cette ou de ces «entités ». D'appartenir à cette communauté depuis de nombreuses années maintenant, je connais par cour cette rhétorique et je sais la déployer.
Cependant, pour des fins de formation, je pense qu'il est beaucoup plus intéressant de soumettre leurs élaborations et leur «cas » à examen en leur faisant dire pourquoi? Leurs fondements de raison - plutôt que de les écraser de notre mépris du haut de notre «science » de la structure!

$\mathrm{Au}$ fond, le plus précieux de ce que j'aimerais vous apporter au cours de ce Séminaire, ce sont non pas des réponses qui sont facilement convertibles en dogmes, toujours difficiles à interroger -, mais des questions, en ce qu'elles ont cette vertu irremplaçable de produire du trou dans le savoir, donc d'aider à penser et à élaborer.

En bien, ces questions, quelles sontelles?

J'en vois cinq fondamentales:

$\rightarrow$ Qu'est-ce que la névrose? Ou: qu'est-ce qu'une névrose

$\rightarrow$ Quelles sont les conditions de la névrose?

$\rightarrow$ Comment classiquement une névrose?

$\rightarrow$ Quels sont les types cliniques de la névrose?

$\rightarrow$ La névrose a-t-elle changé historiquement? Si oui, quelles sont les coordonnées sont les coordonnées de la névrose contemporaine?

Pour essayer de faire avancer cette question, je vais vous soumettre une série d'idées ou d'hypothèses qui, je l'espère, seront dignes de votre attention.

La première idée que je vous propose est la suivante: le trauma et le fantasme sont avant tout des notions «forgées »- en fait, seulement reconstruits - par Freud pour résoudre, dans le champ de la psychanalyse, la question de la cause de la névrose et du symptôme.

La deuxième idée pourrait, elle, se formuler: la cause est relative au discours au sens où Lacan a pu soutenir dans sa «Télévision » que «l'éthique est relative au discours. »

$\mathrm{Au}$ fond, l'hypothèse que je forme est très simple: elle consiste à penser que sur 
le fond d'une théorie de la névrose qu'il hérite de la psychopathologie de son temps, Freud a tenté de conjuguer trois thèses sur la causalité de la névrose et du symptôme. Et plus particulièrement, je voudrais soutenir que les problèmes, les difficultés et les instabilités de la doctrine freudienne du trauma et du fantasme tiennent au fait que ces différentes conceptions ne relèvent pas du même discours, ou plus précisément: relèvent de discours différents, et donc de logiques parfois, voire souvent, discordantes.

1. Je dirai que la première conception et on peut d'une certaine manière la caractériser de pré-freudienne, au sens où Freud en hérite d'une certaine tradition psychopathologique - relève du discours du maître. C'est celle du traumatisme au sens strict, et même de la causalité traumatique, du traumatisme comme cause. Cette cause est censée être un fait réel au sens de l'effectivité. Ce fat réel est une percussion, une collision psychique, l'équivalent psychique, pour ainsi dire, du traumatisme médical: le choc.

Souvenons-nous de ses Etudes sur l'hystérie et du rôle essentiel qu'il y accorde au facteur traumatique dans la pathologie de l'hystérie. Une phrase est, de ce point de vue, tout à fait éclairante: «Au point de vue théorique, écrit Freud, les résultats montrent que le facteur accidentel est, bien au-delà de ce que l'on pensait, déterminant dans la pathologie de l'hystérie. » (Etudes sur l'hystérie, p.1)

Est-il besoin de rappeler que comme son ami Breuer, Freud établissait, au sens strict, un rapport de cause à effet entre le facteur traumatique et les manifestations pathologiques? En effet, dans sa perspective d'alors, chaque accès hystérique suscite une reproduction hallucinatoire de quelques éléments du traumatisme ou de son vécu. Mieux: à l'instar de Breuer, Freud étendait le concept de traumatisme à l'ensemble des phénomènes hystériques, même si ceux-ci paraissent parfois fort lointains.
Autrement dit, les manifestations les plus diverses de l'hystérie pourraient toutes se rattacher à un facteur traumatique: «Evènements survenus dans l'enfance qui ont provoqué, au cours de toutes les années suivantes, un phénomène pathologique plus ou moins grave. » (Etudes sur l'hystérie, p.2)

Que ressort-il de ces énoncés de Freud? Tout simplement une analogie voire une homologie entre hystérie et "névrose traumatique », au point où le concept de «névrose traumatique » en viendra à recouvrir totalement l'ensemble de la pathogénie de la névrose.

$\mathrm{Si}$ j'ai fait ce petit rappel incomplet, je le reconnais - de la première conception de l'hystérie que l'on rencontre chez Freud, c'est pour montrer quoi?

D'abord, la dépendance étroite dans laquelle elle se trouve à l'endroit du discours médical et de la conception de la causalité, mécanique et linéaire, qui y règne. Dans ce discours, en effet, s'il y a de la névrose c'est parce qu'il y a eu un fait réel qui l'a généré, et c'est la fonction de cette cause qui est assignée au traumatisme.

Ensuite, c'est pour souligner l'orientation «victimologique » que cette conception induit. Le névrosé est un traumatisé, donc la victime d'une «mauvaise rencontre ». $\mathrm{Ce}$ qui, bien évidemment, est en contradiction avec l'idée d'un sujet en tant qu'effet actif. Pas étonnant dès lors que ledit sujet lui-même, aidé sans doute par le discours du capitaliste, se mette à se considérer comme sujet à dédommager et à réparer.

Enfin, ne voit-on pas qu'en faisant d'un facteur posé comme accidentel, i.e., contingent, le traumatisme, la cause de l'hystérie, ou bien on sépare la névrose hystérique (dont on ferait une pathologie) de la structure hystérique, ou bien on fait du traumatisme non pas un fait accidentel, contingent, mais un phénomène nécessaire, un fait qui ne peut pas ne pas se produire dans l'histoire d'un sujet. Et alors, la différence entre la névrose hystérique et 
l'assujettissement à la structure hystérique ne serait plus que quantitative!

Mais, le plus grave, sans doute, c'est qu'en faisant du facteur traumatique la cause de la névrose, on accrédite l'idée que dès lors que le sujet entre dans la névrose, en présente la symptomatologie, c'est que l'évènement traumatique s'est réellement produit, et qu'il est nécessaire, pour traiter le sujet, d'en trouver la trace, le souvenir. La voie est ainsi ouverte à la remémoration, sans doute, mais la voie est aussi ouverte à tous les forçages et à toutes les suggestions. Et du coup, à tous les faux souvenirs de la part des patients. Qu'on se souvienne que même de disposer de la catégorie du fantasme, n'a pas mis Freud à l'abri de tels excès. Je fais naturellement allusion à la position qui fut la sienne dans l'analyse de Serguéi Pankejeff alias L'homme aux loups.

2. Venons-en à présent à la deuxième idée que j'ai annoncée. Je dirai, pour faire simple, que cette conception de la causalité de la névrose et du symptôme relevant, selon moi, du discours du maître - la causalité traumatique -, est rapidement supplantée, au moins en partie par une autre conception rattachable quant à elle au discours de l'hystérique. Il n'est pas simple de les distinguer, en raison de leur chevauchement et parfois de leur intrication dans le corpus freudien d'une part, mais aussi, d'autre part, à cause du lien intime entre l'hystérique et le maître, de la fonction structurante de la maîtrise dans l'émergence ou en tout cas le fonctionnement du discours hystérique.

Cette conception de la causalité de la névrose et du symptôme, disons en première approche que c'est celle que les premières hystériques soufflent à l'oreille de Freud. Elle partage avec la première, celle du discours du maitre - on peut tenir la théorie de Charcot pour son paradigme l'idée d'une cause réelle, d'une cause traumatique. Le caractère sexuel dudit traumatisme qu'on trouve chez Charcot luimême vient des hystériques, de ce que
Charcot consent à retenir de ce qu'elles disent sous hypnose et surtout donnent à voir dans les crises hystériques. Il reste que pour Charcot, l'important n'est pas la nature sexuelle du traumatisme mais le fait qu'il y ait eu traumatisme et réminiscence du traumatisme.

Telle que Freud l'articule, quant à lui, la question sexuelle devient centrale, en tout cas pour ce qui concerne ce qu'il appellera les «névroses de transfert», et tout particulièrement pour l'hystérie et l'obsession. Mais, ce sexuel en question, si je puis dire, n'est pas un sexuel de plein exercice. D'où l'expression savoureuse mais très heuristique de Freud: " évènement sexuel présexuel ». Autrement dit, pour Freud il s'agit bien d'une rencontre du sexuel, mais qui survient «trop tôt », pourrait-on dire. Trop tôt non pas par rapport aux aptitudes du sujet à ressentir une excitation sexuelle et des affects liés à elle $\mathrm{du}$ plaisir, du déplaisir (dégoût, par exemple) -, mais trop tôt par rapport à ses capacités de symboliser ce qui lui arrive, à lui conférer un sens phallique donc.

Là où, par l'introduction de la dimension temporelle, certains n'ont vu qu'une forme de développementalisme dans la théorisation de Freud, je vous invite à considérer plutôt l'index qui le met sur la voie de cette forme particulière de causalité - causalité non pas circulaire mais rétroactive - qui est la causalité par aprèscoup (nachträglich).

Le deuxième point qui structure cette deuxième conception freudienne hystorique, pourrait-on dire- de la cause de la névrose et du symptôme, c'est la place qu'elle fait à l'Autre, au désir voire à la jouissance de l'Autre. Rappelez-vous l'équation freudienne: «à fille hystérique, père pervers » -. Cette conception hystorique de la causalité de la névrose se distingue donc particulièrement par le fait d'impliquer l'Autre et la manière dont elle implique cet Autre comme sujet, en faisant par exemple du père l'agent de la séduction.

Ai-je besoin de dire qu'avec le père, nous entrons de plain-pied, non seulement 
dans la découverte de Freud, mais aussi, si l'on peut dire, dans sa subjectivité. La publication récente de la version non expurgée de la Correspondance avec Fliess est là, entre autres, pour en témoigner.

Mais, pour l'élève ou le lecteur de Lacan, il y a aussi et surtout ce que Lacan a identifié, dans Les quatre concepts fondamentaux de la psychanalyse comme le "désir de Freud », désir qu'il invitait à mettre en question pour que la « psychanalyse soit remise sur ses pieds », désir dont la critique le conduira à promouvoir le «désir de l'analyste » comme l'opérateur radical qui est requis pour que psychanalyse il y ait.

Or, ce « désir de Freud », ce fameux «péché originel de la psychanalyse »expression qui a donné son titre à un ouvrage intéressant mais aussi fort déconnant de Gérard Haddad -, vous vous en souvenez sans doute, «ce fait que dans Freud quelque chose n'a jamais été analysé », Lacan le rapporte au père, au désir de «sauver le père », donc peu ou prou à une supposée faute paternelle. C'est la particularité de ce désir qui rendrait raison, selon Lacan, du privilège par lequel Freud a pu trouver « dans le champ de l'expérience de l'inconscient, la voie d'entrée ». Ce désir, s'il se distingue du désir de l'hystérique, celui de «soutenir le désir du père (...) par procuration », lui est aussi lié en tant qu'autre mode d'usage du Nom-duPère.

Le plus important, pour nous, reste quand même, qu'avec cette centralité du père dans la détermination de la névrose et du symptôme névrotique, la voie est ouverte non seulement pour le fantasme, mais aussi pour la causalité langagière, la causalité logique de la névrose et donc pour le sens et la signification des symptômes.

3. La troisième conception qui se rencontre enfin chez Freud - peut-on rigoureusement la tenir pour la causalité relevant du "discours analytique » au sens strict? - Est une sorte d'Aufhebung des deux précédentes, mais pas seulement.
Côté Aufhebung, ou si vous préférez côté synthèse hégélienne, on peut dire qu'elle conserve deux choses des deux premières conceptions:

a) Elle conserve jusqu'à un certain point ce qu'on pourrait appeler le maintien de la réalité, de l'effectivité, de l'historicité du phénomène traumatique, et ce malgré l'abandon de ses neurotica et l'élaboration du concept de fantasme. Là aussi, il suffit de se référer à la construction du cas de $L$ 'homme aux loups par Freud pour être tout à fait édifié.

Mais ce qui frappe en l'occurrence, c'est, au regard de la question du traumatisme réel, la dissymétrie qui apparait dans le traitement par Freud de la névrose hystérique et de la névrose obsessionnelle, notamment si l'on partage le diagnostic freudien de névrose obsessionnelle porté sur le cas de S. Pankejeff. On peut former l'hypothèse que si Freud, dans sa direction de la cure comme dans son articulation du cas, le compte rendu qu'il en a fait, maintient jusqu'au bout la réalité de la scène traumatique, ce n'est pas exclusivement en raison de sa polémique avec Jung ; c'est de manière plus décisive, à cause du fait qu'il s'agit d'une scène primitive et non d'une scène de séduction, et parce que pour ce qui concerne la séduction du jeune Serguéi, ce n'est pas le père mais la sœur et pour la menace de castration, la bonne, Grouschka, qui sont mises en cause dans cette observation. Autrement dit, il n'y avait dans ce cas, aux yeux de Freud, qui relèverait d'une faute du père, rien qui mette en cause le père. Conséquence: nulle manteau de Noé à jeter sur la nudité du père, et nulle inhibition de sa « volonté » de savoir;

b) Ce que cette troisième conception de la causalité de la névrose conserve également des deux premières, c'est que la cause est d'ordre sexuel, que le traumatis me est nécessairement sexuel. Cette perspective, on le sait, marque de son 
empreinte, encore de nos jours, la doctrine communément reçue chez les analystes qui, souvent, excluent du champ du traumatis me tout évènement qui ne soit pas de «nature » sexuelle. Or, même Freud a été conduit, avec les «névroses de guerre » notamment, à élargir sa théorie du trauma.

Il me semble que la question qui nous est posée est donc désormais: qu'ajoute véritablement Freud à ce qu'il a conservé des conceptions hystérique et magistrale de la cause traumatique de la névrose?

J'oserai l'hypothèse que ce qu'il $\mathrm{y}$ ajoute réellement, c'est la notion de fantasme.

Précisons: le fantasme, non pas comme simple doublure imaginaire d'un évènement réel vécu, mais le fantasme en tant que construction imaginaire nouant, symbolique et réel certes, mais qui a la même efficience dans la causation des symptômes qu'un évènement réel: soit une réalité psychique.

En somme, on peut avancer que ce qu'hystérie et hystoire assignent comme trauma, et que le fantasme sature par son scénario, renvoient à ce que, dans le lexique de Lacan, nous appelons communément, la structure. Reste la question difficile mais décisive: pourquoi le fantasme hystérique s'avance-t-il classiquement sous le couvert du traumatisme?

Revenons au fantasme en tant que figure fondamentale de la réalité psychique. Nous savons que Freud y prendra appui pour construire, entre autres, sa doctrine des «fantasmes originaires ». Notion peut-être tombée en désuétude, en tout cas supplantée dans les communautés d'orientation lacanienne parle «fantasme fondamental », le «fantasme dans son usage fondamental », pour reprendre l'exacte formule de Lacan.

J'ai pourtant l'idée que ce terme de «fantasmes originaires » garde tout son prix, et notamment qu'il peut nous guider dans la nécessaire réévaluation de la notion de traumatisme et de «causalité traumatique ».

En effet, pourquoi Freud en vient-il à forger la notion des fantasmes originaires si toujours, et pour chacun, il y a nécessairement un traumatisme réel?

Si je convoque à ce point de mon exposé, ce que Freud a appelé les "fantasmes originaires ", ce n'est bien sûr pas avec l'idée d'en faire une présentation exhaustive. La raison de cette mobilisation est que le détour par cette catégorie freudienne un peu négligée m'a paru nécessaire pour faire un pas supplémentaire en direction du thème de l'année prochaine: l'inconscient.

Du fantasme, il faut bien le dire, on ne rencontre plus dans les publications analytiques que ce qui a trait à sa construction dans la cure, et à sa traversée, qui fut présentée naguère comme le nec plus ultra, le « fin du fin » de la fin de l'analyse.

Il reste que l'importance de ces thématiques ne devrait pas cependant nous faire oublier tout ce que Freud a élaboré par ailleurs autour du fantasme: sa fonction dans l'accès du sujet à la jouissance sexuelle, en particulier dans l'onanisme, ou son rôle dans la formation des symptômes, hystériques notamment (cf. « Les fantasmes hystériques dans leur rapport à la bisexualité »).

Mais la question que je veux ranimer, aujourd'hui, concerne le lien, le rapport du fantasme au trauma. Or, ce lien, c'est en tant qu'il est problématique que Freud y prend appui au moment où il introduit dans sa doctrine les «fantasmes originaires ».

Vous vous souvenez peut-être que c'est dans le mouvement de l'analyse qu'il a consacré, en 1915, à un cas de paranoïa féminine, cas qui présentait à ses yeux l'intérêt d'être «en contradiction avec la théorie psychanalytique », que Freud en est venu à formuler cette idée. Je le cite: «L'observation du commerce amoureux entre les parents est une pièce rarement manquante dans le trésor des fantasmes inconscients qu'on peut découvrir par 
l'analyse chez tous les névrosés, et vraisemblablement chez tous les enfants des hommes. Ces formations fantasmatiques, celle de l'observation du commerce sexuel des parents, celle de la séduction, de la castration, et d'autres, je les appelle fantasmes originaires, et je ferai ailleurs une recherche approfondie sur leur origine ainsi que sur leur rapport à l'expérience vécue individuelle. »(Névrose, psychose et perversion, p. 215)

Tout le monde perçoit, je pense, ce qui, pour Freud, est en question.

Je dirai, pour dégager la logique de sa démarche, que l'expérience de Freud le conduit à faire le constat suivant: certains fantasmes - qu'il ramène à trois fondamentaux (scène primitive, séduction, castration), même s'il laisse la possibilité qu'il y en ait d'autres - certains fantasmes donc se retrouvent chez tous les sujets qui se soumettent à l'expérience analytique.

Freud va même un peu plus loin, de considérer que ces fantasmes se rencontrent chez tous les sujets humains. Manière élégante d'affirmer rien moins que leur universalité d'une part, et d'autre part, d'écarter toute hypothèse pouvant faire de ces fantasmes des artefacts du dispositif analytique.

Or, c'est justement cette universalité supposée des «fantasmes originaires » qui rend suspect, aux yeux de Freud soi-même, le fait qu'à chacun de ces fantasmes corresponde, pour un sujet donné, un évènement réel dans l'expérience vécue individuelle.

N'est-ce pas exorbitant, en effet, de considérer que chaque sujet humain a été réellement l'observateur d'une scène sexuelle entre ses parents, qu'il a été objet de séduction de la part d'un adulte et qu'il a été menacé de castration s'il continuait à transgresser l'interdit de la masturbation?

Si Freud s'est posé ou s'est re-posé le problème du rapport entre le fantasme et l'expérience vécue individuelle, c'est bien parce qu'il a perçu le lien entre le trauma et avec la question de la causalité traumatique de la névrose.

Par ailleurs, s'il y a un problème non seulement théorique mais aussi et surtout clinique, c'est bien parce que la correspondance est surprenante entre les fantasmes dits originaires par Freud et les types de traumatismes que le même Freud a supposé ou entériné à partir des dits de ses analysants comme étant à l'origine des différentes névroses: séduction (hystérie), scène primitive (névrose obsessionnelle), castration (phobie).

Bien sûr, qu'à partir de là les questions se bousculent.

Peut-on, doit-on poser la nécessaire réalité, la nécessaire effectivité d'un évènement traumatique pour qu'il se produise, par après, de la névrose?

Peut-on, doit-on, au contraire, poser que le traumatisme est seulement une fiction nécessaire pour donner «forme », pas du tout épique d'ailleurs, à ce qui s'opère de la structure?

$\mathrm{Ou}$ enfin, peut-on et doit-on conjuguer ces deux hypothèses et postuler différents modes, différents types d'articulation de la contingence du trauma et de la nécessité du fantasme qui, à l'occasion, supplée au trauma lorsqu'il manque?

Difficile, évidemment, de répondre frontalement à des questions aussi difficiles.

Mettons de côté, si vous le voulez bien, la «pitrerie darwinienne »de Freud, je veux dire sa postulation selon laquelle l'enfant apporterait en naissant des schémas phylogénétiques, «schémas qui, semblables à des «catégories » philosophiques, ont pour rôle de « classer» les impressions qu'apporte ensuite la vie. » (In «L'homme aux loups », Cinq psychanalyses, p. 418)

Freud poursuit mais pour introduire à une tout autre logique: «Je suis enclin à penser qu'ils (les schémas) sont des précipités de l'histoire de la civilisation humaine. Le complexe d'Edipe, qui embrasse les rapports de l'enfant aux parents, est l'un d'eux; il en est, de fait, 
l'exemple le mieux connu. Là où les évènements ne s'adaptent pas au schéma héréditaire, ceux-ci subissent dans l'imagination un remaniement, travail qu'il serait certes profitable de suivre dans le détail. Ce sont justement ces cas-là qui sont propres à nous montrer l'indépendante existence du schéma. Nous avons souvent l'occasion d'observer que le schéma triomphe de l'expérience individuelle (...). » (In «L'Homme aux loups », idem, p. 418)

Ainsi, Freud corrige-t-il la biologie par l'histoire, la phylogenèse par le complexe d'Edipe.

La question qui se pose à nous est donc désormais: pouvons-nous nous satisfaire d'une telle solution? L'Edipe ne relève-t-il pas davantage du mythe que de l'histoire? Pourquoi cette correction n'a - $t$ elle pas définitivement remise le traumatisme à sa vraie place dans les structures freudiennes de la névrose?

Je dirai juste, pour ma part, que si l'on met en cause le ressort évolutionnis te de la thèse de Freud, il ne nous reste plus que la structure. Et c'est sans doute pourquoi Lacan s'est tourné vers ce paradigme pour trouver une solution viable aux problèmes hérités de Freud.

En prenant comme point de départ, non pas l'expérience individuelle, mais la structure de langage qui est la cause de ce qu'il y a du sujet dans le réel, autrement dit en tournant le dos à l'empirisme, on voit aussitôt se dessiner une perspective autre.

Dans cette autre perspective, il apparait que c'est la prise du sujet parlant dans le langage - c'est, je crois, l'une des valeurs à donner à la thèse de Lacan sur le caractère traumatique du signifiant - qui le confronte non seulement à des énigmes ou à de l'asémantique, à la bêtise du signifia nt, mais à des réels, c'est-à-dire à des impossibilités logiques:

$\rightarrow \quad$ Le réel du sexe qui, avant d'être formulé par Lacan comme l'inexistence du rapport sexuel dans l'inconscient, a été approché par
Freud comme l'impossibilité à laquelle est soumise tout sujet de ne pouvoir appartenir aux deux sexes à la fois;

$\rightarrow \quad$ Le réel du temps qui articule l'impossibilité d'exister en deçà de sa conception et au-delà de sa mort;

$\rightarrow \quad$ Le réel du père et des générations qui renvoie à l'impossibilité d'appartenir en même temps à sa propre génération et à celle de ses parents, et qui formule mezzo voce la loi fondamentale qui structure les sociétés humaines: l'interdit de l'inceste.

Qui ne voit qu'en somme, c'est le même dire qui se déduit des trois fantasmes, autrement dit qu'il n'y a de fantasme que de castration ( $v s$ menace de castration)?

Alors, me direz-vous, comment rendre raison de la névrose sans la nécessité du trauma? Et à partir de quel concept de l'inconscient?

Sans doute que même un autre séminaire ne suffirait pas pour poser, développer et discuter ce que je vais introduire à présent. $\mathrm{Au}$ fond, l'idée même est simple, et pourrait se formuler: il y a, a minima, deux théories de la névrose.

La freudienne, dans laquelle la notion de traumatisme est importante, voire indispensable, en raison de sa surdétermination parle « désir de Freud » et le fantasme hystérique d'une part, et par sa dépendance à l'endroit d'un concept de l'inconscient qui ne tient " qu'à supposer le Nom-du-Père ». A côté ou au-delà de la névrose selon Freud, il y a, si je puis dire, la «névrose lacanienne ». Non pas au sens ironique où Lacan a pu parler d'une «psychose lacanienne », à propos du cas Gérard Primeau évoqué à son Séminaire, mais au sens d'une véritable doctrine de la névrose qui articule celle-ci d'une manière différente voire opposée à elle de Freud. Mais, pour mettre au jour cette doctrine lacanienne de la névrose, il est nécessaire de surmonter un préjugé tenace, celui de l'homologie des thèses freudiennes et 
lacaniennes, et de se donner comme principe de lecture la surprenante formule prononcée par Lacan à l' " Ouverture de la Section Clinique »: «L'inconscient donc, n'est pas de Freud, il faut bien que je le dise, il est de Lacan ». Ce que Lacan explicite, en ajoutant: «On ne peut pas dire que l'inconscient soit par lui (Freud) vraiment isolé, isolé comme je le fais par la fonction que j'ai appelée du symbolique, et qui est pointée dans la notion de signifiant. »

Je ne reprendrai pas ici, bien évidemment, ce que j'ai développé sur ce point dans le cadre de notre Séminaire d'Ecole de 2005-2006 à Toulouse. J'aimerais simplement faire valoir qu'il y a une théorie lacanienne de la névrose non triviale, irréductible à la freudienne, et que nous gagnerions à ranimer.

La première articulation de cette doctrine de la névrose, Lacan la produit dans son séminaire du 27 juin 1956.

Que dit-elle?

D'abord, que la névrose en tant qu'elle est l'affaire d'un sujet et non une pathologie individuelle, la névrose donc est « une question posée par le sujet au niveau de son existence même. " Et Lacan de reprendre, de manière plus serrée, ce qu'il a déjà énoncé dans son Séminaire sur "Les structures freudiennes des psychoses ».

«Cette question prend dans l'hystérie les formes suivantes - Qu'est-ce que c'est que d'avoir le sexe que j'ai? Qu'est-ce que veut dire avoir un sexe? Qu'est-ce que veut dire que je puisse même me poser la question?»

\section{(...)}

Si la névrose se rapporte au niveau de l'existence, elle s'y rapporte de façon plus dramatique encore dans la névrose obsessionnelle, où il s'agit non seulement du rapport du sujet à son sexe, mais de son rapport au fait même d'exister. C'est ainsi que se situent comme obsessionnelles les questions - Qu'est-ce que c'est que d'exister? Comment suis-je par rapport à celui que je suis sans l'être, puisque je puis en quelque sorte me dispenser de lui, prendre assez de distance pour me concevoir comme mort?» (La relation d'objet, p. 391)

Lacan y ajoute que non seulement que la névrose est une question - et une question fermée, autant que le sujet ne sait pas qu'il est dans cette question-, mais qu'au fond, c'est une langue et un texte, un texte dans lequel nous nous retrouvons au moyen d'un certain nombre de structures.

Il s'en suit que « dans notre effort de déchiffrement, nous devons suivre ce qui a été noué effectivement dans le texte de la névrose. Or, ce texte est soumis à l'utilisation, dans la situation actuelle, d'un élément du passé du sujet comme élément signifiant. Voici une des formes les plus claires de l'x de la condensation. Lorsque nous abordons les éléments signifiants du texte, nous ne pouvons donc nous abstraire du fait qu'il se décompose en deux termes situés en deux points très éloignés dans l'histoire du sujet, et pourtant il nous faut bien résoudre les choses dans le mode d'organisation où elles sont actuellement. C'est ce qui nous commande de chercher les lois propres à la solution de chacun de ces discours organisés, selon le mode desquels les névroses se présentent à nous. » (J. Lacan, La relation d'objet, p.393)

On voit combien nous sommes loin de toute psychopathologie et de ce que toute visée cathartique requiert comme type de causalité. Non seulement «l'inconscient est structuré comme un langage », mais l'inconscient est langage, parce qu'il est le symbolique comme tel, le rond $\mathrm{du}$ symbolique de la structure borroméenne du sujet, ce qui restera irréductiblement l'Autre pour tout sujet de la parole.

La deuxième réélaboration par Lacan de la névrose, nous en trouvons les articulations dans son Séminaire «D'un Autre à l'autre » (1968-1969) et tout particulièrement dans la séance du 18 juin 1969.

Si précédemment dans le Séminaire, il reprend l'idée de la névrose comme «question fermée », le pas accompli désormais est d'introduire, à la surprise générale, l'implication de départ du sujet 
supposé savoir dans la névrose, qu'elle soit hystérique ou obsessionnelle.

En quoi me direz-vous? En ceci justement que l'hystérique, de mémoire d'analyste, est captivée par «la femme supposée savoir ce qu'elle veut », de même que l'obsessionnel s'en remet - servitude volontaire? - Au «maître supposé savoir ce qu'il veut ». Ce que Lacan met magistralement en évidence, c'est que cet usage de la femme par l'un et du maître par l'autre n'a qu'une seule fonction: colmater le vide de l'Autre, le vide de la structure inconsciente.

Lacan en déduit que cette structure constituante du sujet supposé savoir fait du névrosé rien moins qu'une sorte d' « analysant naturel », assignant ainsi à l'analyste l'opération de lui attendue : rien de moins, comme le disait excellemment Michel Bousseyroux que de «détacher le névrosé de cette supposition dont il affuble, s'il est hystérique, la femme, et s'il est obsessionnel, le maitre, pour qu'il puisse par lui-même savoir ce qu'il veut, soit, savoir ce qu'est son désir et l'insatisfaction , s'il est hystérique, ou l'impossible s'il est obsessionnel.» (In, L'inconscient lacanien, p.16)

Peut-on éviter la question qui, je suppose, ne manque pas de se poser pour chacun, à savoir: quelle utilité le concept de trauma peut-il avoir pour nous afin de cerner et surtout d'opérer, en tant qu'analyste, sur la névrose ainsi définie?

Osons répondre, pour conclure, à la question: «Pourquoi y a-t-il de la névrose et pas plutôt que rien? »

Après le parcours que nous avons accompli, on voit bien - du moins je l'espère - que l'on ne peut y répondre comme Angelus Silesius en disant que « La névrose est sans pourquoi »-, justement parce que, et que ce ne peut être simplement, comme semble le suggérer la doxa analytique, parce que le sujet a vécu dans son enfance une expérience traumatique d'ordre sexuel.

En effet, non seulement il n'est guère vérifiable que tous les sujets ont été partout et depuis toujours soumis à de telles expériences, ni que tous les sujets qui y ont été soumis en fassent trauma, mais il est des évènements authentiquement traumatiques pour certains sujets qui ne génèrent nulle névrose.

Dans sa «Question préliminaire à tout traitement possible de la psychose », Lacan indiquait déjà, et de façon très claire, que la condition du sujet, névrose ou psychose, dépend de ce qui se déroule dans l'Autre, détermination symbolique donc par l'Autre qui précède le sujet et sans doute contraint toutes les expériences à venir dans l'existence de ce dernier.

En effet, si nous prenons au sérieux la définition lacanienne de la névrose, « la névrose comme question », il faudrait peutêtre ajouter: mais une question dont le sujet a fait le choix d'y répondre par le père, le Nom-du-Père, le symptôme - père. Amour de ce père comme constituant l'armature même de l'hystérie ou confusion du père et du maitre et attente de sa mort à l'instar de l'esclave du mythe de Hegel, meurtre du père comme régulateur de son désir pour l'obsessionnel. C'est ce désir, insatisfait ou impossible, qui imprime donc sa marque et sa couleur à la cause, s'il est vrai comme le soutient Lacan, dans L'angoisse, qu'il n'y a pas de cause d'avant le désir.

Dès lors, on peut avancer que s'il y a de la névrose, c'est-à-dire du sujet divisé aux prises avec l'impossible du rapport sexuel, c'est moins parce que telle ou telle expérience a été par lui vécue, mais parce que d'avant toute expérience de séduction, de scène primitive ou de menace de castration, le sujet a été confronté au seul «traumatisme » de structure auquel nul ne saurait échapper, celui lié au fait de la rencontre du signifiant, du sexe, du trou de l'Autre, et pour chaque névrosé, en tout cas Lacan le suggère, d'une certaine figure de l'Autre : le «parent traumatique ».

Pour conclure réellement à présent, permettez-moi de dire que contrairement à ce que l'on a tendance à croire, la psychanalyse, non seulement est loin de s'être émancipée des savoirs sur le fonds 
desquels elle s'est constituée, mais des élaborations décisives développées en son sein - par Lacan, en particulier -restent ensevelies sous le fatras de nos idées reçues. Peut-être découvrirons-nous, l'année prochaine, que finalement, l'inconscient, le signifiant-maître de notre discours, est un concept beaucoup plus neuf que nous n'osons le penser!

Data de submissão: 03/07/2017

Data de aceite: 17/08/2016 